\section{Colitis ulcerosa \\ Anämie erhöht das Risiko für Hospitalisierung}

\begin{abstract}
Anämie kommt bei Colitis ulcerosa häufig vor, behandelt werden die Patienten aber zu selten, beklagt Prof. Dr. Andreas Stallmach, Direktor der Klinik für Innere Medizin am Uniklinikum Jena, beim diesjährigen Gastro Update in Wiesbaden.
\end{abstract}

Die Anämie ist die häufigste systemische Komplikation von chronisch entzündlichen Darmerkrankungen (CED). Eine aktuelle Längsschnittstudie an einem US-amerikanischen Referenzzentrum hat eine Rate von $45 \%$ bei Colitis ulcerosa-Patienten ergeben.

Die hohe Prävalenz wird durch die Behandlungszahlen jedoch nicht abgebildet. „Weniger als die Hälfte der Patienten erhält eine spezifische Therapie“, beklagte Prof. Dr. Andreas Stallmach beim diesjährigen Gastro Update in Wiesbaden. Der Direktor der Klinik für Innere Medizin am Uniklinikum Jena betonte, dass die CED-assoziierte Anämie eine konsequente Diagnostik und Therapie erfordert. „Eine persistierende Anämie ist ein unabhängiger Risikofaktor für Hospitalisierungen und chirurgische Eingriffe.“ Umgekehrt seien Patienten mit kompliziertem Verlauf - stattgehabter OP oder Behandlung mit Immunsuppressiva oder Biologika - und auch Frauen besonders gefährdet, eine Anämie zu entwickeln.

Eine häufige Ursache der CED assoziierten Anämie ist Eisenmangel. Die Diagnose einer Eisenmangelanämie anhand des Ferritinwertes kann jedoch Probleme bereiten: „Ferritin ist ein Akutphase-Protein und kann bei einer gleichzeitig bestehenden Inflammation falsch hoch sein“, erinnerte Stallmach. Um einen Eisenmangel nicht zu übersehen, kann im Fall einer
Entzündung bei Ferritinwerten $>30 \mathrm{ng} / \mathrm{ml}$ der Transferrinrezeptor-Ferritin-Index (TfR-F) bestimmt werden. TfR-F-Werte $>2$ zeigen ein Eisendefizit an.

Zur Behandlung bei Eisenmangelanämie sehen die Leitlinien eine parenterale Therapie vor, sofern der Hb-Wert $<10$ g/dl liegt oder eine erhöhte Krankheitsaktivität oder eine Intoleranz gegen orale Eisenpräparate besteht. Die Versorgungsrealität in Deutschland sieht aber anders aus: Nach einer von Stallmach vorgestellten Studie erhielten von 183 CED-Patienten mit Anämie in Schwerpunktpraxen und Krankenhausambulanzen gerade einmal $44 \%$ eine anämiespezifische Therapie.

\section{Orale Eisensubstitution}

Nach Einschätzung von Stallmach würde eine "gut verträgliche und effektive orale Eisensubstitution das Therapiemanagement deutlich vereinfachen“. Tatsächlich wird oral appliziertes Eisen aber nur zu 5-10\% resorbiert, und bis zu 50\% der $\mathrm{Pa}-$ tienten klagen über gastrointestinale $\mathrm{Ne}$ benwirkungen. Mit einem EisenmaltoseKomplex zeichnet sich jetzt eine erfolgversprechendere orale Behandlungsoption ab: Eisenmaltol führte in einer Studie zur CED-assoziierten Anämie zu einer raschen und klinisch relevanten Verbesserung des Hb-Wertes, ähnlich wie sie sonst bei parenteraler Eisensubstitution erzielt wird.

(bs)

\title{
INFOS IM INTERNET
}

Die aktuelle Leitlinie zu Diagnostik und Therapie bei Colitis ulcerosa ist verfügbar unter: www.dgvs.de/leitlinien/colitis-ulcerosa

Das Kompetenznetz Darmerkrankungen bietet Infos zu CED. Hier gibt es Fachinformationen für Ärzte sowie laienverständliche Beiträge: www.kompetenznetz-ced.de

Ratgeber für Patienten mit Colitis ulcerosa stehen auf der Seite der Gastroliga unter "unser Service" zur Verfügung: www.gastroliga.de

Alle Folgen der Sommer-Akademie finden Sie in der App der „Ärzte Zeitung“

(auch im Web unter www.aerztezeitung.de)

\section{ÜBRIGENS}

...scheint ein Mikrobiomaustausch bei Pouchitis zu helfen. Bei drei der fünf behandelten Colitis ulcerosa-Patienten verschwand die Entzündung komplett, bei einem weiteren stellte sich eine Besserung ein.

Am J Gastroenterol 2016; 111:441-4439.

...ist Schwerpunkt des Crohn \& Colitis Tages 2016 das Thema Freundschaft, Partnerschaft und Arbeitsalltag. Aktionen finden vom 24. September bis zum 23. Oktober statt.

...konnten Forscher einen Mechanismus identifizieren, durch den die Störung der Darmbarriere bei chronisch entzündlichen Darmerkrankungen erklärt werden kann. Das aktivierte Protein Rho-A sorgt wohl für die Stabilität des Epithelzellverbands . (grz)

J Clin Invest 2016; online 11. Januar

Punkte sammeln in der Sommerakademie mit e.Med

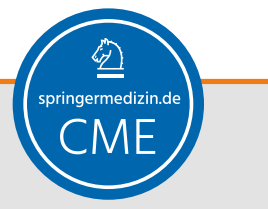

Auch 2016 haben wir in der Sommerakademie ausgewählte CME-Kurse für Ihre hausärztliche Fortbildung zusammengestellt.

Zum Thema "Colitis ulcerosa" finden Sie unter www.springermedizin.de/sommerakademie aktuell den Kurs

,Biomarker für chronisch entzündliche Erkrankungen"

Zur Teilnahme benötigen Sie das e.Med-Abo, mit dem Sie Zugang zu allen Inhalten auf Springer-Medizin.de erhalten - kombiniert mit einer gedruckten Fachzeitschrift Ihrer Wahl. Als e.Med-Abonnent haben Sie nicht nur Zugriff auf die Sommerakademie, Sie können das komplette CME-Kursangebot von SpringerMedizin.de nutzen. Hier finden Sie aktuell rund 550 CME-zertifizierte Fortbildungskurse aus allen medizinischen Fachrichtungen! Damit ist CME.SpringerMedizin.de Deutschlands erste Adresse für Online-CMEFortbildung.

Nutzen Sie die Sommerzeit, um CME-Punkte zu sammeln und um sich schnell und effizient fortzubilden. Testen Sie CME.SpringerMedizin.de 30 Tage lang kostenlos und unverbindlich mit dem e.Med-Abo: www.springermedizin.de/eMed 